\title{
Servicios ecosistémicos como soporte para la gestión de sistemas socioecológicos: aplicación en agroecosistemas
}

\author{
Ecosystem Services as support for the Management of socio- \\ ecological systems: application in agroecosystems
}

\section{Serviços Ecossistemicos como suporte para a gestão dos sistemas sócio-ecológicos: aplicação em agroecossistemas}

\author{
Clara I Caro-Caro ${ }^{1 *}$; Marco A Torres-Mora ${ }^{2}$ \\ 1 Bióloga MSc, \\ 2 Biólogo PhD \\ * Grupo de investigación GIGAS, Instituto de Ciencias Ambientales de la Orinoquia Colombiana, Facultad de Ciencias \\ Básicas e Ingeniería, Universidad de los Llanos, Villavicencio, Colombia \\ Email: clarainescaro@unillanos.edu.co
}

Recibido: diciembre 12 de $2014 \quad$ Aprobado: noviembre 17 de 2015

\begin{abstract}
Resumen
Con el propósito de responder a la pregunta ¿los nuevos enfoques interdisciplinares de servicios ecosistémicos y de sistemas socioecológicos son pertinentes para la gestión sostenible tanto de sistemas naturales como de agroecosistemas? se hace una revisión de la evolución de los conceptos servicios ecosistémicos y sistemas socioecológicos, de los avances temáticos con análisis crítico, y con mayor detalle en los estudios sobre agroecosistemas realizados en Colombia. Se examinan controversias y enfoques diferenciales desde la ecología y economía para sintetizar en la delimitación de tres ejes centrales a desarrollar en trabajos con énfasis local: la comprensión y manejo de la incertidumbre, los métodos para identificar, cualificar y cuantificar los servicios ecosistémicos y sus relaciones con la biodiversidad (trades-offs, dis-servicios, sinergias) y el análisis particular y ajustado al contexto de los servicios ecosistémicos culturales.
\end{abstract}

Palabras clave: biodiversidad, Orinoquia, altillanura, compensaciones, gestión ambiental, sostenibilidad

\begin{abstract}
With the purpose to answer question the new interdisciplinary approaches to ecosystem services and socio-ecological systems are relevant for the sustainable management of both natural systems as agroecosystem?, A review of the evolving concepts of ecosystem services and socio-ecological systems is made, and thematic developments with critical analysis in greater detail in studies on agro made in Colombia. Disputes and differential approaches are examined from ecology and economics to synthesize in delimiting three core to develop work with local emphasis: understanding and managing uncertainty and methods to identify, qualify and quantify ecosystem services and their relationship with biodiversity (trades - offs, dis- service, synergies) and the particular analysis and adjusted to the context of cultural ecosystem services.
\end{abstract}


Key words: biodiversity, Orinoco, highthills, trades-off, environmental management, sustainability

\begin{abstract}
Resumo
A fim de responder à pergunta as novas abordagens interdisciplinares de serviços ecossistemicos e de sistemas sócioecológicos são relevantes para a gestão sustentável de ambos os sistemas naturais e agrossistemas?, se faz avaliação da evolução dos conceitos serviços ecossistêmicos é sistemas socio ecologicos, dos avancos tematicos com análise crítica, e em maior detalhe nos estudos sobre agro ecossistemas feitos na Colômbia. Se discute abordagens diferenciais de ecologia e economia para sintetizar na delimitação de três eixos centrais para desenvolver trabalhos com ênfase nas áreas locais de compreensão e manejo da incerteza, os métodos para identificar, qualificar e quantificar os serviços dos ecossistemas e suas relações biodiversidade (comércios-offs, sinergias, dis-service) e a análise especial e adaptadas ao contexto dos serviços ecossistemicos culturais.
\end{abstract}

Palavras-chave: biodiversidade, Orinoco, planaltos, remuneração, gestão ambiental, sustentabilidade

\section{Introducción}

A lo largo de la historia los humanos se han preguntado y dado explicaciones sobre la naturaleza, su interrelación con ella y su manejo. En occidente el concepto de naturaleza evoluciona desde Platón quien en su obra Critias alude a la deforestación y sus efectos (400 a d c) y Aristóteles en su filosofía primera de la naturaleza (60 a d.c.); pasa por la visión creacionista, los estudios naturalistas y de distribución de especies con Linneo (1788), Humboldt (1807), Darwin (1872) y el dominio de la ciencia reduccionista -mecanicista antropocéntrica hasta el siglo XIX. En los pueblos ancestrales de América, África y Australia, en ese mismo período se manifiesta la relación hombre-naturaleza de manera integral sin compartimentalización ni separación tangibles del homo y lo "otro", la naturaleza. La importancia de los recursos naturales se reconoce en el contexto mundial desde el siglo XIX, en 1830, con el trabajo realizado por Malthus, acerca del crecimiento poblacional (Malthus, 1963). Durante el siglo XX, los nuevos aportes en dinámica poblacional, en investigaciones del medio ambiente junto con las crisis ambientales originadas por la revolución industrial y las posguerras derivaron en una necesidad de entender la interrelación sociedad-ambiente-recursos naturales y plantear nuevos paradigmas a la demanda y manejo de los recursos naturales, ante un mundo globalizado, con el desarrollo de dos grandes ejes de investigación, la ecología de ecosistemas y el ambientalismo. Como punto de partida se menciona a Pinchoth quien en 1910 fundamentó principios y leyes para áreas de parques naturales en Estados Unidos.

Surge así la sostenibilidad como paradigma emergente, que de acuerdo a Carrizosa Umaña (1992) es un concepto que integra y expresa la necesidad de una transición desde las agendas puramente sociales o ambientales hacia una agenda sistemática e integrada (Larraín y Dávila, 2004). Su origen se remontó al Club de
Roma en 1968, prosiguió en 1972 en Estocolmo (King et al., 1973), se oficializó por las Naciones Unidas en "Nuestro Futuro común" (Brundtland, 1987), se concretó en la Cumbre de Naciones Unidas sobre Medio Ambiente y Desarrollo en Rio de Janeiro (ONU, 1992), con seguimientos en el 2002 en la Cumbre Mundial sobre el Desarrollo Sostenible (ONU, 2002), en el 2012 en la Conferencia de Naciones Unidas sobre el Desarrollo Sustentable, Rio+20 (ONU, 2012). De manera consecuente se abordó el concepto de capital natural, término usado por primera vez por Schumacher (1973) (citado por Gómez-Baggethun et al., 2010) y que alude a las reservas de la naturaleza en materiales o información que producen un flujo sostenible de valiosos bienes y servicios útiles o renta natural a lo largo del tiempo y expresa la idea de acerca de que ningún sistema económico organizado puede mantenerse sin tener en cuenta el flujo de los recursos renovables y no renovables (Costanza y Daly, 1992; Prugh, et al., 1999). Asimismo, a finales del siglo XX e inició del XXI se impulsó el concepto de servicios ecosistémicos definidos como un amplio rango de condiciones y procesos a través de los cuales los ecosistemas naturales y las especies que hacen parte de ellos ayudan a sostener la vida humana y se aplican en decisiones de gestión y política (Daily, 1997; Millennium Ecosystem Asessement, MEA, 2003; Cork et al., 2006; Ash et al., 2010). El concepto de sistemas socio-ecológicos surgió en los años 90 para aludir a sistemas caracterizados por cambios discontinuos próximos a sus valores críticos en sus umbrales y el efecto de la resiliencia en la organización y la salud del mismo, así como por su enfoque interdisciplinario entre las ciencias naturales y las ciencias sociales (Perrings, 1994; Folke, 2006; Epstein et al., 2013). De forma convergente con los nuevos paradigmas descritos, el concepto de biodiversidad se sigue discutiendo en relación con los servicios ecosistémicos y los conflictos que puedan generar las contraposiciones o incompatibilidades entre servicios 
ecosistémicos, los trade-offs, (MEA, 2005) y las decisiones de gestión de los mismos (Rincón-Ruiz et al., 2014; Martín-López et al., 2014).

Se puede plantear así que la evolución conceptual desde la noción de naturaleza hasta los servicios ecosistémicos ha sido consecuente con los cambios en paradigmas que han surgido para responder las preguntas referidas a la interacción sociedad - naturaleza - ambiente (Figura 1). Como principales influyentes en estas disertaciones se encuentran, desde la filosofía de Aristóteles que postuló la separación del hombre del resto de los seres vivos y continua hasta los naturalistas en el siglo XIX (Humboldt, 2011). Vendrá el ambientalismo, la corriente de raíces anglosajonas que denuncia la contaminación química en las ciudades y posteriormente en los campos agrícolas, encabezada por Rachel Carson (1962). Con la prevalencia de la economía neoclásica en los años 70 del siglo XX aparece la sostenibilidad en el discurso político y en la academia, por ejemplo en los términos capital natural y desarrollo sostenible como enfoques. La globalización como modelo económico incide igualmente desde los años 70 y se expresa esencialmente en los objetivos del milenio (MEA, 2003, 2005) y en las metodologías para evaluar la biodiversidad y sus servicios (The economics of ecosystems and biodiversity, TEEB 2010)

Ante la pregunta ¿los nuevos enfoques interdisciplinares de servicios ecosistémicos y de sistemas socioecológicos son pertinentes para la gestión sostenible tanto de sistemas naturales como de agroecosistemas?, se desarrollan tres capítulos a saber: evolución conceptual de servicios ecosistémicos y sistemas so- cioecológicos; situación en avances temáticos, con énfasis en agroecosistemas en Colombia; controversias acerca del concepto servicios ecosistémicos, su aplicación y relaciones con la biodiversidad. Para finalizar se presentan las perspectivas a abordar en los temas expuestos y la propuesta del enfoque integrador entre la ecología y la economía ecológica, a través de la valoración integral de los servicios ecosistémicos, que posibilitaría su aplicación en la toma de decisiones acerca del manejo sostenible de la biodiversidad y los agroecosistemas.

\section{Evolución de los conceptos servicios ecosistémicos y sistemas socio-ecológicos}

Se aborda el concepto de servicios ecosistémicos en su evolución en el siglo XX, sin desconocer las raíces griegas y el desarrollo posterior que tuvo hasta el siglo XIX, en la ciencia occidental. En la década de 1950 se reconoce la dependencia humana del ambiente, entendido como capital natural (Schumacher,1973; Vanderwalle et al., 2008). Posteriormente con el enfoque de sistemas ecológicos a partir de la termodinámica (Odum, 1969; Margalef, 1993) se utilizó la denominación de recursos naturales para todo lo que provenía de los ecosistemas y la biósfera en general y hacia parte fundamental del mantenimiento de las sociedades humanas, en esencia los materiales o insumos directos o indirectos del entorno natural, no creados por la actividad humana y que son escasos (Cooper, 1975), en tanto que Márquez (1996) se refirió a los de bienes y servicios ecológicos fundamentales que cumplen funciones de soporte vital para la sociedad, característica de los ecosistemas estratégicos. Los recursos na-

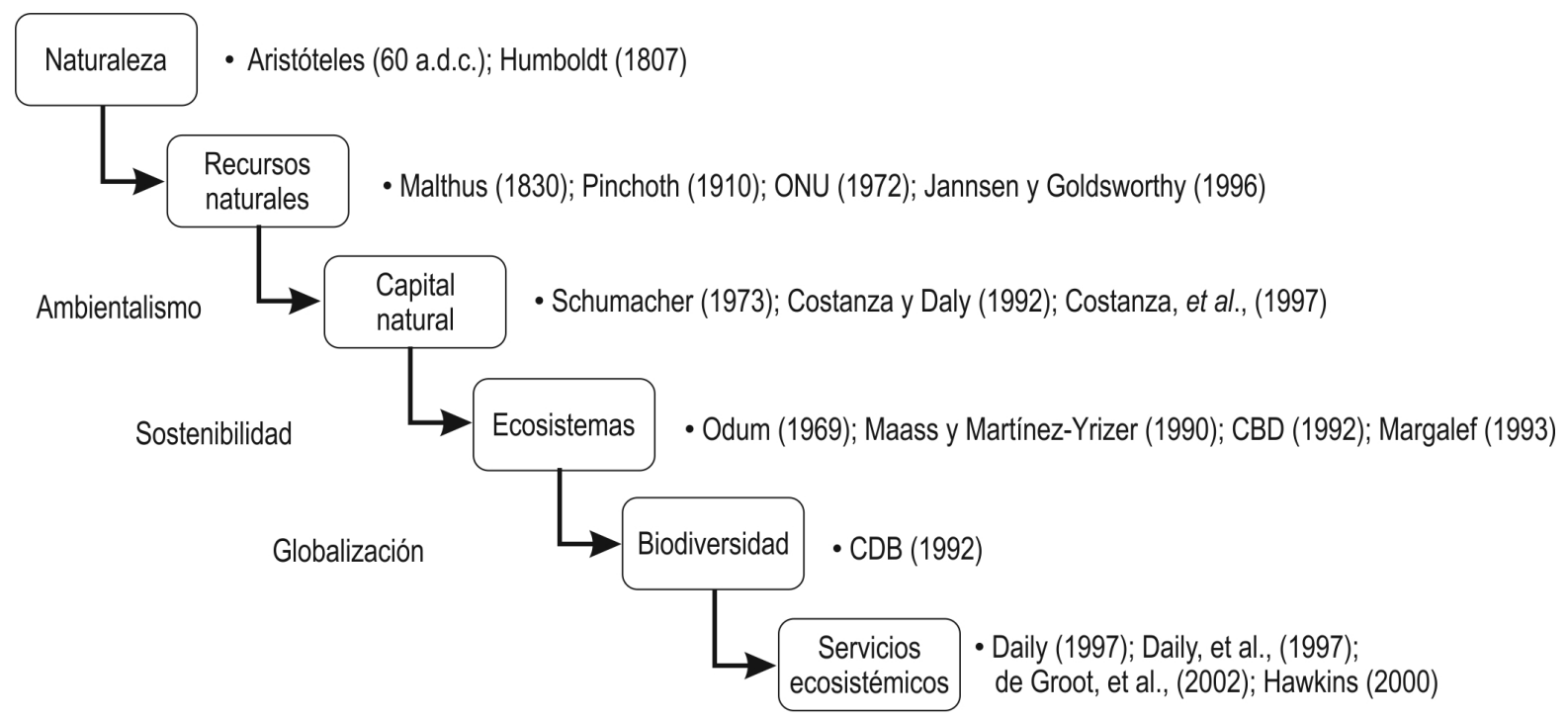

Figura 1. Evolución conceptual del enfoque: servicios ecosistémicos 
turales se reconocen como económicamente útiles en la producción o el consumo, bien sea como factores de producción ó como bienes que pueden ser objeto de comercio internacional World Trade Organization (WTO, 2010), como variables de una influencia poderosa asociada a conflictos violentos y a intereses de seguridad en Estados débiles, cuyo colapso puede ser evitado, si éstos se distribuyen equitativamente (Motesharrei et al., 2014, Douglas y Alie, 2014). Se plantean para su manejo dos aspectos importantes, investigaciones interdisciplinarias que aporten creatividad y articulación de actores sociales y grupos de interesados (Janssen y Goldsworthy, 1996; Humphreys, 2005; Anastasopoulu et al., 2007).

Desde la perspectiva ecológica los recursos naturales entran a las sociedades humanas como bienes o servicios, según cumplan una función, antes de la crisis económica de finales de la década del 70. Inicialmente se hizo bajo el enfoque de capital natural, definido para indicar todas las reservas de la naturaleza en materiales o información que producen un flujo sostenible de valiosos bienes y servicios útiles o renta natural a lo largo del tiempo y que abarca interacciones que determinan integridad y resiliencia ecológica de los ecosistemas (Costanza y Daily, 1992; Costanza et al., 1997; Gómez-Baggethun y de Groot, 2007). Otros autores plantean la necesidad de preservar, conservar y renovar el capital natural para el desarrollo económico actual, con políticas efectivas que tomen en cuenta los costos naturales en todas las decisiones de tipo económico y donde la biodiversidad y el conocimiento ancestral sean componentes fundamentales del mismo (Grima et al., 2003; MEA, 2005; Conabio, 2006; González et al., 2008). En tanto investigadores como MacDonald et al. (1999) concluyen que el concepto de capital natural puede ser un vehículo útil para cambiar las actitudes hacia un mayor reconocimiento de la contribución de los recursos naturales para el bienestar humano.

\section{Servicios ecosistémicos: conceptualización y enfoques}

La importancia de los recursos naturales se reconoce en el contexto mundial desde el siglo XIX con el trabajo de Malthus, A summary view of the Principle of Population, impreso publicado en 1830 y en el período 1905- 1910 Gifford Pinchoth fundamenta y aplica principios y leyes para áreas de parques naturales en Estados Unidos como protección de estos recursos. Holdren y Ehrlich (1974) definieron los servicios ya enunciados en el capital natural como las funciones de los servicios de carácter público proporcionados por el ambiente global que no pueden reemplazarse por tecnología ahora o en un futuro previsible y que incluyen, entre otros, el control de plagas, la polinización, la regulación del clima. En tanto que para Ehrlich y Mooney (1983) los servicios ecosistémicos corresponden a las funciones ecológicas del ecosistema, definición que desarrolla Daily (1997) a un amplio rango de condiciones y procesos, a través de los cuales, los ecosistemas naturales y las especies que hacen parte de ellos ayudan a sostener la vida humana, por ejemplo como fuente de materias primas, de alimento $y$ de productos medicinales. Diversos autores en este contexto ecológico destacan la importancia de relacionarlos con la capacidad de aquellos que satisfacen directa o indirectamente las necesidades, usos y preferencias humanas (Daily et al., 1997; de Groot et al., 2002; Hawkins, 2003; Kremen, 2005). El concepto de servicios ecosistémicos evoluciona desde la ecología con criterios de valoración denominándose bienes y servicios ambientales, esenciales en el funcionamiento de los ecosistemas, el suministro de bases primarias para la producción de alimentos y el bienestar humano (Costanza et al., 1997; MEA, 2005; TEEB, 2010).

En el contexto global, de manera reciente el informe de ecosistemas del milenio - MEA (2003, 2005) plantea para los servicios ecosistémicos un marco de referencia conceptual, metodológico y de aplicación a la toma de decisiones. En su definición de servicios ecosistémicos precisa "son los beneficios directos e indirectos que la humanidad recibe de la biodiversidad" y los agrupa en cuatro categorías: de aprovisionamiento ó bienes y productos brindados por los ecosistemas tales como los alimentos, el agua, los recursos genéticos, los productos forestales; de regulación que atañen a los beneficios obtenidos de la regulación de los procesos ecosistémicos e inciden en el clima, las inundaciones, la calidad del agua; culturales definidos como los bienes no materiales obtenidos de los ecosistemas: el enriquecimiento espiritual, el desarrollo cognitivo, la reflexión, la recreación y las experiencias estéticas; de soporte o apoyo que se refieren a procesos ecológicos necesarios para la provisión y existencia de los demás servicios ecosistémicos, tales como la producción primaria, la formación del suelo y el ciclado de nutrientes.

Una vez impulsado el uso del concepto en la arena política y académica (MEA, 2005), diversos autores abordan tópicos que difieren, convergen o complementan lo propuesto; así por ejemplo Boyd y Banzhaf (2007) precisan "los servicios de los ecosistemas no son los beneficios humanos obtenidos de los ecosistemas, son los componentes ecológicos directamente consumidos o disfrutados para producir bienestar humano" por lo cual procesos indirectos y funciones no son servicios ecosistémicos; Balvanera y Cotler (2007) expresan que "servicios ecosistémicos" y "servicios am- 
bientales" pueden ser utilizados sin distinción, aunque el primero se refiere al ecosistema y sus interacciones y los beneficios a los seres humanos, en tanto que el segundo alude al concepto de "ambiente", que no explicita interacciones para proveer servicios y se utiliza por tomadores de decisiones. Al respecto la propuesta del MADS (2012) los diferencia y acepta para servicios ambientales lo enunciado por Constanza et al., (1997) se refieren en general al flujo de materiales, energía e información del capital natural, combinado con capital humano o manufacturado para el bien humano, y la amplia al suministro de recursos ambientales o saneamiento ambiental prestados por industrias y organizaciones sociales, como los servicios de alcantarillado, recogida y disposición de basuras, saneamiento y servicios similares, al igual que servicios de reducción de emisiones de los vehículos y servicios de reducción del ruido, entre otros, más no están necesariamente relacionados con los procesos y funciones de los ecosistemas, como sí lo están los servicios ecosistémicos. Fisher et al., (2009) plantean que corresponden al resultado de una función de las interacciones complejas entre éstas y su entorno abiótico, al uso y aprovechamiento de estos patrones complejos y a las diversas percepciones de los beneficiarios.

En referencia al bienestar humano como elemento central de la definición de servicios ecosistémicos (Boyd y Banzhaf, 2007; Fisher et al., 2009), se llama a profundizar en un aspecto: ¿cómo relacionar el incremento de medidas para aumentar el bienestar humano con el descenso de las condiciones de los ecosistemas? (Raudsepp-Hearne et al., 2010; Ash, et al., 2010); al respecto Lele et al., (2013) plantean que "la contribución de la naturaleza biótica para el bienestar humano es reconocido y subvalorado, lo que resulta en la destrucción de los ecosistemas".

En el tema de categorización Fisher et al., (2009) consideran que además de los componentes del sistema ecológico de interés se deben incluir los contextos sociales y políticos en los que los servicios de los ecosistemas están siendo investigados o utilizados. En tanto que Rositano et al., (2012), al comparar diversas clasificaciones a partir de Daily (1997), destacan que una de las principales diferencias es la falta de consenso acerca de la categoría donde está el servicio de mantenimiento de la biodiversidad; se avanza incluso hasta distinguir los servicios ecosistémicos de los servicios del paisaje al involucrar éstos el componente social (Lamarque et al., 2011; Vallés-Planells et al., 2014). En este sentido se menciona la importancia de identificar y monitorear los servicios ecosistémicos con métodos lo más concretos posibles sin sesgar su análisis (Díaz et al., 2006; Rincón-Ruiz et al., 2014) o cuantificar los servicios ecológicos con unidades de servicio biofísico y no económico (Egoh et al., 2007); y de tener enfoques interdisciplinares en su estudio y análisis (Costanza y Kubiszewski, 2012; Spash y Aslakse, 2012; Barnaud y Antona, 2014).

Se puede sintetizar la conceptualización de servicios ecosistémicos en tres enfoques: ecológico, económico y ecológico-económico (Vandewalle et al., 2008). En la tabla 1 se presenta una modificación a esta propuesta, incorporando las temáticas específicas, aunque de manera reciente surge el enfoque diferencial para los servicios culturales (Martín-López et al., 2012a; Rositano et al., 2012). En esta misma línea se presentan postulados en las opciones de cuantificación ecológica y valoración cualitativa (Egoh et al., 2007; Lamarque et al., 2011; Vilardy et al., 2012), de cuantificación ecológica y valoración económica de estos servicios (Martín-López et al., 2012b, 2014; Rincón-Ruiz et al., 2014) a través de métodos de la economía ecológica y la economía ambiental. Asimismo como lo expresan Rositano y Ferraro (2014), los servicios ecosistémicos proveen un marco sencillo para la toma de decisiones, que puede parametrizarse y cuantificarse fácilmente.

\section{Biodiversidad y servicios ecosistémicos: relaciones e intercambios}

En este aspecto varios autores plantean la importancia de evaluar el rol de la biodiversidad en el suministro de los servicios (Carpenter et al., 2009, de Groot et al., 2010, Martín-López y Montes, 2011) con estudios que permitan establecer de que forma los componentes de la biodiversidad y mediante que procesos de relacionamiento aportan al suministro de los servicios ecosistémicos (Kremen, 2005; Burkhard et al., 2009; Harrison et al., 2010; Lavelle et al., 2014). En el mismo sentido se reconoce la complementariedad entre biodiversidad y servicios ecosistémicos (Haines-Young y Potschi, 2009); Lamarque et al., 2011). Adicionalmente se utilizan los nombres de unidad suministradora de servicios y de unidad beneficiaria (Fisher et al., 2009) que se sintetizan en el término proveedor de servicios para denotar poblaciones, comunidades, grupos funcionales, caracteres funcionales, tipos de hábitats y paisajes con capacidad de generar servicios a la sociedad (MartínLópez y Montes, 2011). Así distintas investigaciones evidencian como las disposiciones espaciales de las unidades funcionales, los hábitats, los elementos del paisaje y el uso de la tierra influyen significativamente en la generación de servicios ambientales (de Groot et al., 2010; Syrbe and Watz, 2012; Andrade et al., 2014).

El concepto biodiversidad y su relación con el concepto de servicios ecosistémicos se puede explicar en 
Tabla 1. Evolución del concepto servicios ecosistémicos: enfoques

\begin{tabular}{|c|c|c|c|}
\hline Enfoque & Definición & Temas desarrollados & Referencia \\
\hline Ecológico & $\begin{array}{l}\text { "un amplio rango de } \\
\text { condiciones y procesos } \\
\text { a través de los cuales los } \\
\text { ecosistemas naturales y las } \\
\text { especies que hacen parte de } \\
\text { ellos ayudan a sostener la } \\
\text { vida humana" (Daily, 1997) }\end{array}$ & $\begin{array}{l}\text { Servicios públicos y ambiente global. } \\
\text { Biodiversidad y conservación } \\
\text { Bienes y servicios ecológicos. } \\
\text { Servicios ecosistémicos y } \\
\text { bienestar humano. } \\
\text { Servicios ecosistémicos y } \\
\text { cuantificación ecológica. } \\
\text { Biodiversidad y Servicios ecosistémicos. }\end{array}$ & $\begin{array}{l}\text { Holdren y Ehrlich, } 1974 \\
\text { Ehrlich y Mooney, } 1983 \\
\text { Márquez, } 1996 \\
\text { Daily, 1997; Kremen, } 2005 \\
\text { Vandewalle et al., } 2008 \\
\text { Martín-López y Montes, } 2011\end{array}$ \\
\hline Económico & $\begin{array}{l}\text { "Los servicios del ecosistema } \\
\text { consisten en flujos de } \\
\text { materiales, energía e } \\
\text { información de reservas } \\
\text { de capital natural que se } \\
\text { combinan con los servicios } \\
\text { de capital manufacturados } \\
\text { y humanos para producir } \\
\text { el bienestar humano" } \\
\text { (Costanza et al., 1997). }\end{array}$ & $\begin{array}{l}\text { Bienes y servicios ambientales } \\
\text { Capital natural y bienestar humano. } \\
\text { Capital natural y resiliencia } \\
\text { Servicios ecosistémicos: } \\
\text { son bienes públicos. } \\
\text { Servicios ecosistémicos oferta } \\
\text { (valoración ecológica) demanda } \\
\text { (valoración sociocultural y monetaria). }\end{array}$ & $\begin{array}{l}\text { Costanza y Daly, } 1992 \\
\text { Costanza y Folke, } 1997 \\
\text { Costanza et al., } 1997 \\
\text { Hawkins, 2003 } \\
\text { TEEB (2010) }\end{array}$ \\
\hline $\begin{array}{l}\text { Ecológico } \\
\text { Económico }\end{array}$ & $\begin{array}{l}\text { "los servicios ecosistémicos } \\
\text { son los beneficios directos } \\
\text { e indirectos que la } \\
\text { humanidad recibe de la } \\
\text { biodiversidad" (MEA, 2005) }\end{array}$ & $\begin{array}{l}\text { Funciones: regulación, hábitat, } \\
\text { producción, información. } \\
\text { Marco conceptual y metodológico } \\
\text { de servicios ecosistémicos. } \\
\text { Valoración de ecosistemas y servicios } \\
\text { ecosistémicos - herramienta pragmática. } \\
\text { Capital natural y servicios } \\
\text { ecosistémicos: sostenibilidad. } \\
\text { Gestión de la biodiversidad: garantía } \\
\text { de servicios ecosistémicos. }\end{array}$ & $\begin{array}{l}\text { de Groot et al., } 2002 \\
\text { MEA, 2003, } 2005 \\
\text { Gómez-Baggethun y } \\
\text { de Groot, } 2007 \\
\text { Montes, } 2007 \\
\text { Martín-López y Montes, } \\
\text { 2011; MADS, 2012; } \\
\text { Rincón-Ruiz et al., 2014 }\end{array}$ \\
\hline
\end{tabular}

varios niveles: como soporte para procesos claves, al afectar directamente el suministro de servicios ecosistémicos y al constituir en sí misma un bien valorado (Mace et al., 2012). Así, las interacciones entre biodiversidad y servicios ecosistémicos pueden expresarse en diferentes vías: adoptar la forma de "trade-offs ó compromisos (es decir, donde la promoción de un servicio reduce la oferta de otro servicio); de una situación ganar-ganar (cuando un solo paquete de gestión mejora el suministro de diversos servicios); o las sinergias, donde el uso simultáneo de los servicios aumenta o deprime tanto más que si se utilizan de forma independiente" (MEA, 2005). Al respecto Martínez-Alier et al., (1998) expresa que los trade-offs entre servicios ecosistémicos demandados por la sociedad son inconmensurables (no pueden ser agregados a un valor monetario o biofísico). La gestión de los servicios ecosistémicos puede alterar la cantidad o la calidad de los mismos lo que lleva a considerar la importancia de abordar sus efectos en escalas espaciales y temporales (Rodríguez et al., 2006; Rincón-Ruiz et al., 2014). Los trades-off pueden ser temporales, aquellos que implican beneficios ahora y costos a largo plazo; espaciales, los que generan un beneficio local pero tienen costos en otro lugar (local, regional o global); e interpersonales, que ocurren cuando unos actores disfrutan de determinado servicio en tanto que otro grupo de actores sociales no lo puede hacer Martín-López y Montes (2011).

En torno a estas relaciones entre la biodiversidad y sus servicios, las tendencias de estudios proponen desde la funcionalidad de los ecosistemas, investigaciones acerca de las numerosas interacciones que pueden ocurrir entre múltiples servicios ecosistémicos (Bennet et al., 2009; Martín-López et al., 2012a; Maskell et al., 2013), como escenarios de enfoques de sostenibilidad, de política de focos sociales y de organizaciones sociales 
dominantes, en los cuales es probable que se obvien procesos sociales y ecológicos vinculados que funcionan en escalas espacio temporales (Cork et al., 2006; Martín-López y Montes, 2011). También se destaca la necesidad de identificar y cualificar las relaciones de sinergias y trade-offs entre servicios como base para la toma de decisiones políticas (Rincón-Ruiz et al., 2014; Martín-López et al., 2012a, 2014) y de los factores demográficos-económicos, sociopolíticos, culturales como impulsores de cambio global de los ecosistemas y sus servicios (Nelson et al., 2006; Anastasopoulu et al., 2008; MADS, 2012).

Las alternativas sobre manejo de la biodiversidad, como fuente de los servicios de los ecosistemas y no como servicio en sí, no siempre son comparables puesto que los trade-offs varian de acuerdo a la priorización de servicios ecosistémicos efectuada; ante ello surge la necesidad de contabilizar los factores que predicen los trade-offs (MEA 2005; TEEB, 2010). En el contexto económico Díaz et al., (2006) plantean que la pérdida de servicios ecosistémicos - dependientes de la biodiversidad - probablemente acentúa "la desigualdad y la marginación de los sectores más vulnerables de la sociedad, al disminuir su acceso a los tangibles básicos para una vida saludable y reducir su libertad de elección y acción", por ello la conclusión de de Groot et al., (2010) es pertinente "las inversiones en la conservación, restauración y uso sostenible de los ecosistemas se ven cada vez más como una situación que genera beneficio ecológico, social y económico sustancial".

\section{Sistemas socioecológicos o socioecosistemas, aproximación conceptual}

El concepto de ecosistema como unidad integral constituye un enfoque unificador para el análisis de la naturaleza desde dos ámbitos, la ecología y la gestión ambiental. En la evolución del mismo desde la propuesta de Tansley en 1935, se llega en el siglo XX a una definición holística que incorpora las variables espaciotemporales :"Un complejo dinámico de comunidades vegetales, animales y de microorganismos en su medio no viviente que interactúan como una unidad funcional" (CDB, 1994). Pero esta sigue apartando a las sociedades humanas como componente y transformador de lo restante, lo vivo y lo no vivo, de la naturaleza. En la década de los 90, Márquez (1996) esboza el término de ecosistemas estratégicos, entendidos como aquellos importantes para la sociedad porque le prestan una serie de bienes y servicios más allá de las funciones ecológicas propias de su dinámica natural; en esta misma línea se pueden citar a Daily et al., (2000), de Groot et al., (2002), Spash y Aslaksen (2012).

De otro lado, a partir de la misma década y hasta el presente diversos autores reconocen los ecosistemas como proveedores de bienes y servicios ambientales, entendidos como funciones de los ecosistemas pero con un enfoque de capital natural o de importancia para el bienestar de la sociedad (Constanza y Daily, 1992; Costanza y Folke, 1997; Rodríguez-Labajos y Martínez-Alier, 2013) o servicios ecosistémicos en la concepción de los beneficios recibidos por la sociedad y soportados en los ecosistemas como expresión de la biodiversidad (Daily ,1997; Kremen, 2005; Gómez-Baggethun y de Groot, 2007; TEEB, 2010; RincónRuiz, et al., 2014).

En Colombia la política nacional cambia el concepto ecosistema del Convenio de Diversidad Biológica, a la significación de gestión de la biodiversidad que incluye el manejo integral de sistemas ecológicos y sociales íntimamente relacionados, dependiente de la conservación como atributo emergente - resultado de la interacción entre sistemas de preservación, de restauración, de uso sostenible y de construcción de conocimiento e información (MAVDS, 2012).

Puede considerarse como un punto de partida del concepto de sistema socioecológico el planteado por Martínez-Alier et al., (1998) los ecosistemas "son sistemas complejos reflexivos" que incluyen el estudio de las dimensiones humanas del cambio ecológico y de las transformaciones de las percepciones ambientales humanas. Desde ahí se presentan múltiples enfoques y aplicaciones del concepto, en distintas escalas espaciales. Anderies et al., (2004) se refieren a "un sistema ecológico estrechamente vinculados con uno o más sistemas sociales", de carácter complejo y sujeto a variaciones espacio temporales donde las diferencias de percepción de los actores inciden en la toma de decisiones. Los autores plantean el concepto de robustez para calificar la capacidad de respuesta de estos sistemas, así "un sistema socioecológico es robusto si se impide que los sistemas ecológicos en los se basa, de trasladarse a un nuevo dominio de atracción, no puedan apoyar una población humana o induzcan a una transición que cause sufrimiento humano a largo plazo". En años siguientes el concepto de resiliencia social reemplaza el concepto de robustez ampliándose a las interacciones a múltiples escalas espaciales y temporales y a la condición de incertidumbre (Janssen y Ostrom, 2006; Ostrom, 2009; Martín-López et al., 2009, 2011, 2014). Andrade et al., (2011) postulan el reconocimiento del territorio como un sistema socioecológico, en donde interesan los componentes 
sociales o ecológicos individuales y son de gran importancia las interacciones entre éstos. Con incidencia en la toma de decisiones y la gestión ambiental, MADS (2012) define los sistemas socioecológicos como "unidades con expresión espacial concreta, determinada por las relaciones entre sus subsistemas, donde la discernimiento de sus límites espaciales y funcionales dependerá de los objetivos de la investigación, del entendimiento de los problemas sociales y de las soluciones deseadas".

La toma de decisiones políticas y de gestión en última instancia es un asunto de elección de la sociedad, sin embargo bajo el enfoque ecosistémico esa decisión tiene que basarse en el discernimiento de los límites biofísicos que inciden en los procesos ecológicos y de las escalas espaciales y temporales en los que operan (Haines-Young y Potschin, 2009), que conlleva a una valoración integral de los servicios ecosistémicos con técnicas monetarias y no monetarias (Christie et al., 2012; Martín-López et al., 2012a; Rincón-Ruiz et. al., 2014, Torres et al., 2014). Se sugiere que una manera de delimitar estos servicios, partiendo del componente social, es reconocer ciertos caracteres que les imprimen identidad singular a grupos sociales o comunidades, entre ellos diferencias culturales, étnicas, religiosas o políticas, de aislamiento o acceso a redes de información, que pueden, en un momento dado, configurar un sistema social "separado" y "reconocible" (Rincón-Ruiz et al., 2014).

Como apoyo para las decisiones de manejo en los sistemas socioecológicos, el concepto de servicios ecosistémicos es útil como herramienta de integración de los enfoques ecológico y económico y permite aproximarse al entendimiento del vínculo de interdependencia entre las sociedades que los demandan en función de su bienestar y el funcionamiento de los ecosistemas (sistemas ecológicos), como proveedores de manera directa o indirecta.

\section{Avances en sistemas socioecológicos- agroecosistemas}

Los agroecosistemas se pueden definir como sistemas ecológicos asociados a variables socioeconómicas, que tienen como fin la producción de bienes y servicios de importancia económica y constituyen esencialmente un ecosistema modificado (Sarandón, 2014); actualmente se abordan como sistemas socioecológicos y se valoran sus servicios, en esencia, son ecosistemas productivos de origen humano (MADS, 2012). Bustamante (2014) expresa que los agroecosistemas son fuente potencial de ampliación de la diversidad biológica, de diversificación de la producción y de efectos de complementariedad y sinergismos con sistemas naturales, en donde se pueden incorporar los intereses y percepciones de los actores sociales (TapeIla, 2012).

En Colombia se puede partir de reconocer que el empobrecimiento biótico constituye un legado de escala superior en el territorio, donde los cambios y transformaciones en la biodiversidad dependen de impactos acumulados en el territorio, producto de la huella ecológica histórica (Andrade y Castro, 2012) y de tener en cuenta cómo las nuevas actividades económicas, como en el caso de los Llanos Orientales, se convierten en motores de cambio que inciden en la cobertura y manejo de las áreas. En el socioecosistema de la ciénaga Grande de Santa Martha (Vilardy et al., 2012), usan un paradigma cualitativo - inductivo y de perspectiva holística aplicado a una cualificación de los servicios ecosistémicos de lagunas y zonas de pesca como agroecosistemas. En la Orinoquia, Andrade-Pérez et al., (2013) proponen para la altillanura un modelo de adaptación de la agricultura intensiva al sistema ecológico a través de un equilibrio económico y ecológico, en la escala del paisaje; posteriormente encuentran que la agroindustria puede hacerse transformando una parte del territorio reteniendo elementos de ecosistemas naturales sin llegar a una pérdida neta de biodiversidad (Andrade et al., 2014). También en los Llanos Orientales pero en sabanas inundables Mora-Fernández y Peñuela-Recio (2013) desarrollan indicadores de salud del ecosistema en función del contexto y el conocimiento y manejo tradicionales, abordando la biodiversidad y los servicios ecosistémicos en sistemas ganaderos criollos.

\section{Sinergia - trade-offs en sistemas socioecológicos agrícolas en Colombia}

La sinergia entre servicios ecosistémicos que se relacionan entre si se manifiestan en una evolución conjunta positiva, lo que implica que un aumento en el suministro de uno de ellos implica un aumento en el suministro de los otros; por ejemplo el mantenimiento de la fertilidad del suelo propicia el reciclado de nutrientes y la productividad primaria, que a su vez aumentan la capacidad de almacenar carbono y por ende su capacidad de regulación climática (Martín-López et al., 2012b). La sinergia entre servicios ecosistémicos depende del mantenimiento de la multifuncionalidad de los ecosistemas; es mayor en los sistemas naturales que en los agroecosistemas monofuncionales, que alteran la estabilidad de las zonas rurales y su capacidad de provisión de diversos servicios ecosistémicos (García Llorente et al., 2012). 
Respecto a la Orinoquia colombiana se evidencia el impacto en el cambio de la cobertura de uso del suelo, medido en la transición de las diferentes categorías hacia cultivos de palma de aceite (Elaeis sp.) y plantaciones forestales, así en el intervalo 1987 - 2000 éstos se establecen sistemáticamente en los herbazales de tierra firme e inundables y en el bosque inundable, mientras que en el segundo intervalo 2000-2007 se ubican específicamente en áreas previamente ocupadas por pastos (IAvH, 2013). Algunos autores citan cambios negativos asociados a los cultivos de palma de aceite: en lo social la concentración de la propiedad al estar asociada a economía de escala (Ruiz, 2007), en tanto que Goebertus (2008) y Arosa (2015) mencionan el aumento de migración y con ello el incremento de conflictos sociales, y de otra parte en términos de violencia o desplazamiento en zonas de producción (Benavides, 2011). Asimismo, se argumenta que altera la biodiversidad ecosistémica por cambio de uso y pérdida de hábitats de fauna y la flora nativas y por la inclusión de suelos dedicados previamente a la producción de alimentos (Ochoa y Chávez, 2010); de manera reciente se denota el deterioro crítico del servicio ecosistémico cultural en el municipio de Villanueva, con la llegada de este cultivo intensivo (Fernández, 2013).

En contraposición, se reporta que las plantaciones de palma de aceite (Elaeis sp.) que reemplazan cultivos transitorios tienen impactos positivos como la generación de empleo, estimado en 1 empleo/10 ha de cultivo (Mosquera y García 2005); su aporte continuo de materia orgánica al suelo (León et al., 2006); en servicios ecosistémicos de microclima y de polinización (Labarca et al., 2009), la relación carbono almacenado y el balance neto de la emisión de gases de efecto invernadero (Henson et al., 2012).

En otros agroecosistemas, como los cafetales se reporta su servicio como matriz favorable para la diversidad de hormigas (Rivera y Ambrecht, 2005), como paisajes culturales (Rivera, 2008). Para áreas de mayor cobertura se tienen algunos estudios, entre ellos para la cuenca del río La Vieja donde se identifican 13 bienes y servicios de cuales sólo cinco son prioritarios para los actores locales porque aportan a su bienestar y desarrollo (sombra, rompe viento, fijación de nitrógeno, belleza escénica y control de la erosión) (Cardona, 2011). En la altillanura Lavelle et al., (2014) investigan acerca de la biodiversidad y los servicios ecosistémicos asociados al recurso suelo, en grandes sistemas de producción, pasturas mejoradas, cultivos anuales (arroz, maíz y soja), plantaciones de palma de aceite y caucho, contrastándolos con la sabana seminatural; reportan que el manejo de la fertilización química es uno de los principales impulsores de los procesos del suelo; la biodiversidad y los ciclos biogeoquímicos heredados de la sabana semi-natural original (materia orgánica, reservas de nutrientes) son características esenciales para entender el estado del sistema y comprender las trayectorias actuales y futuras de los cambios en los ecosistemas, entre otros; Sanabria et al., (2014) en la misma zona de trabajo proponen indicadores basados en comunidades de hormigas, como herramienta comprender los impactos del cambio en el uso de la tierra y una mejor gestión de los agricultores.

A pesar de tener argumentos de relaciones negativas entre biodiversidad y servicios ecosistémicos en sistemas socioecológicos de agricultura intensiva es necesario considerar las variables espacio temporales y el accionar institucional para tomar decisiones concertadas y acertadas; dado que el suministro de servicios ecosistémicos, en los niveles correspondientes para los beneficiarios, requiere que las políticas sectoriales y la gestión de los ecosistemas estén integrado con las estrategias de conservación de la biodiversidad (Reyers et al., 2012). Al respecto el estudio de Harrison et al., (2010), con datos de Europa hasta 2008, visibiliza la importancia de agroecosistemas especialmente en los servicios de aprovisionamiento vitales (alimentos y madera), mientras que los ecosistemas semi-naturales son claves en provisión de recursos genéticos y en servicios culturales (valores estéticos y sentido de apropiación).

En el marco tendencial de sostenibilidad para los sistemas socioecológicos agrarios cobran gran importancia las conclusiones y recomendaciones hechas por Lavelle et al., (2014): la organización del territorio agrícola debe partir de considerar la importancia de la red de bosques de galería y de corrientes y cuerpos de agua; el monitoreo de la calidad del suelo y de la provisión de servicios de los ecosistemas debe hacerse a escala de paisaje a lo largo del tiempo, para detectar degradación del suelo, evitar impactos irreversibles, aumentar la comprensión de los determinantes de los servicios ecosistémicos y permitir el diseño de paisajes agrícolas ecoeficientes, especialmente aplicables a la situación actual de crecimiento agroindustrial acelerado en la altillanura colombiana.

\section{Revisiones de conceptos y su desarrollo. Controversias y enfoques diferenciales}

Entre las múltiples revisiones se destacan la realizada por Balvanera y Cotler (2007), quienes identifican cuatro grupos de publicaciones orientadas a servicios ambientales y servicios ecosistémicos: marco conceptuales y metodológicos, estudios de caso, valoración 
económica y la incidencia en la toma de decisiones. En esta misma década Skourtos et al., (2010) concluyen que la mezcla de métodos y la puesta en común de los datos parece ser el único camino a seguir en la valoración de servicios ecositémicos, destacando el potencial de la investigación interdisciplinaria sistemática y formal que integre los puntos de vista, los métodos y los datos extraídos de las ciencias naturales y las ciencias sociales. Balvanera et al., (2009) hacen una descripción sintética de trabajos en servicios ecosistémicos tanto como en servicios ambientales, expresando la urgencia de tener acciones integrales que permitan maximizar el mantenimiento de los distintos servicios ecosistémicos.

Harrison et al., (2010) reportan para Europa un incremento en la demanda de servicios ecosistémicos de agroecosistemas y de ecosistemas de recreación y ecoturismo, en tanto que disminuyen la producción ganadera, de agua dulce, la pesca de captura y alimentos silvestres. Para Latinoamérica se encuentra que el mayor énfasis de investigación se direcciona a servicios de provisión: carbono y agua (Balvanera et al., 2012). Rositano et al. (2012) encuentran 1250 referencias para los términos servicios ecológicos y servicios ecosistémicos y hacen un análisis de las clasificaciones propuestas. En Colombia como punto de apoyo para la formulación de la política de gestión de la biodiversidad y los servicios ecosistémicos se hace una actualización de los trabajos realizados en el tema (MADS, 2012).

En el orden internacional Trabucchi et al., (2012) revisan investigaciones enfocadas a la restauración, especialmente métodos, destacando la necesidad de lograr su estandarización. Costanza y Kubisszewski (2012) compilan y analizan la producción académica desde 1983 hasta el 2011; registran 2386 autores y destacan la interdisciplinariedad como enfoque al encontrar que sólo el $12 \%$ no tiene coautores.

En revisión de estudios de agroecosistemas Van den Belt y Blake (2014) aluden a la necesidad de realizar investigaciones que cuantifiquen la prestación de múltiples servicios y los compromisos y las sinergias entre ellos. Howe et al., (2014) examinan estudios de tradesoffs, período 2000-2013, concluyendo que se pueden lograr sinergias y cambiar el enfoque ganar-ganar si se contabilizan los factores que determinan un trade-off por ejemplo, interés privado, grupos de interesados locales. Gómez-Baggethun et al., (2010) trabajan en la evolución histórica del concepto de servicios ecosistémicos y encuentran que desde el enfoque económi$\mathrm{co}$, ha tenido lugar de forma creciente un proceso de mercantilización, es decir la reproducción de la lógica del mercado, junto con su ideología subyacente y las estructuras institucionales, para abordar los problemas ambientales.

Llevada al extremo, la visión de los servicios ecosistémicos podría llegar a considerar a los ecosistemas únicamente por su utilidad directa a las sociedades, lo que conlleva a poner en peligro el mantenimiento de los ecosistemas en su integralidad (Montes 2007). Norgaard (2010) expresa literalmente "la metáfora de la naturaleza como una acción que proporciona un flujo de servicios es insuficiente. El enfoque de servicios de los ecosistemas puede ser parte de una solución más amplia, pero su dominio en la caracterización de nuestra situación y la solución nos está cegando a los valores ecológicos, complejidades económicas y políticas de los desafíos que enfrentamos en realidad". A su vez, Balvanera et al., (2011) concluyen que el concepto de servicios ecosistémicos presenta ventajas claras que explican su uso creciente y refieren a los vacíos de información en un tema tan amplio. Sin embargo, Lamarque et al., (2011) proponen usar el término "servicios ecosistémicos" en el contexto de la conservación de la biodiversidad al ser su objetivo original y la dependendencia de componentes de la biodiversidad para tener dichos servicios y el término " los servicios del paisaje " para uso del suelo y planificación, ya que se basa en los patrones y prácticas de uso de la tierra y que está abierto a los insumos humanos.

Sobre el enfoque económico (Díaz et al., 2006) expresan que el desarrollo económico que no considera los efectos sobre los servicios de los ecosistemas puede disminuir la calidad de vida de poblaciones vulnerables, incluso si otros segmentos de la sociedad se benefician; las valoraciones económicas no son adecuadas en la gestión de la conservación, precisamente en las estrategias de manejo de hábitat que afectan a la prestación de servicios y la conservación de la biodiversidad (Egoh et al., 2007). En aspectos de valoración económica se registran críticas como las de Spangeberg y Settele (2010) quienes afirman que el valor calculado de los ecosistemas y sus servicios no es una figura robusta, varía con el método de valoración aplicado y por ello no es posible calcular "objetivamente" el valor de los servicios ecosistémicos. En este aspecto McAfee (2012) argumenta "... la contradicción entre el desarrollo y la conservación observado en pago de servicios ecosistémicos es inevitable en proyectos enmarcados en la lógica asocial de la economía clásica".

Con relación a agroecosistemas Rositano et al., (2012) plantean dos aspectos claves a considerar en los servicios de los ecosistemas, los cuantitativos como la 
determinación de la relación entre la cantidad y calidad de los servicios provistos y la condición de los ecosistemas que los proveen y los cualitativos como la percepción de distintos actores sociales sobre el estado actual de la provisión de servicios; en esta misma línea se debe tener en cuenta el factor tiempo como determinante en la gestión de los recursos naturales y considerar la huella ecológica histórica de los impactos acumulados en el territorio (Andrade y Castro, 2012).

De manera reciente en el enfoque de servicios ecosistémicos se abordan situaciones como las expresadas por Crossman et al., (2013) "una evaluación de los servicios de los ecosistemas puede ayudar a construir un puente entre el desarrollo y las comunidades ambientales mediante información confiable y robusta sobre los vínculos entre la gestión del ecosistema y la consecución de los objetivos económicos y sociales"; por Balvanera et al., (2011) quienes aducen que el uso del término fuera de contexto, sin enfatizar la necesidad de mantener ecosistemas sanos y diversos para asegurar su capacidad de generar beneficios a las sociedades, ha llevado a una caricatura del término; por Lele et al., (2013) que analizan los inconvenientes del concepto y su aplicación debido a la confusión sobre las funciones de los ecosistemas y la biodiversidad, la omisión de los dis-servicios, los trade-offs con el componente abiótico y un marco de valoración económica usado en la medición del bienestar humano; por Laurans y Mermet (2014) quienes concluyen que se requiere un uso claro del enfoque en la valoración económica de los servicios ecosistémicos para superar la actual "brecha de implementación" del mismo junto con algunos problemas conceptuales y metodológicos subyacentes. A manera de síntesis de lo expuesto, el artículo de Barnaud y Antona (2014) es el más representativo en resumen de controversias; cita las incertidumbres científicas acerca de relaciones causales inherentes a la producción de servicios ecosistémicos; las múltiples interpretaciones dadas al concepto en razón de las diferentes representaciones de las relaciones sociedad-naturaleza, las divergencias acerca de la noción de valor en sí mismo - con notaciones cuantitativas, cualitativas y de percepción - y valoración del servicio ecosistémico, los conflictos de intereses así como los desacuerdos en torno a los instrumentos de política y de gestión referidos al concepto.

\section{Perspectivas}

La comunidad científica, desde las ciencias naturales, sociales y ambientales evidencia un creciente interés en los conceptos de sistemas socioecológicos y de servicios ecosistémicos, aproximadamente en los últimos 20 años, dada la particularidad de tener la opción de aplicarlos en decisiones políticas y de gestión ambiental (Chee, 2004; Troy y Wilson, 2006; MADS, 2012) y como respuesta ante la actual demanda creciente de recursos provenientes de la biodiversidad (Schneiders et al., 2012; Vilardy et al., 2012; Partidario y Gomes, 2013). Los retos expuestos por de Groot et al., (2010) para integrar los servicios ecosistémicos en la planificación, gestión y diseño del territorio siguen vigentes, tales como el entendimiento y cuantificación de los servicios provistos por los ecosistemas, la valoración y uso de los servicios ecosistémicos en análisis de tradeoffs y su uso en la planificación y la gestión del uso sostenible de los servicios ecosistémicos.

Si bien en el caso de Colombia se tiene una política (MADS, 2012) que inserta los nuevos enfoques interdisciplinares de servicios ecosistémicos y de sistemas socioecológicos y reconoce la importancia de la gestión de la biodiversidad como base para lograr este flujo de servicios ecosistémicos y la recepción de los beneficios a los actores sociales del territorio y se cuenta con una metodología de valoración integral (Rincón-Ruiz et al., 2014) correspondiente a la nueva consideración de tres tipos de servicios ecosistémicos: de provisión, de regulación y de culturales, al entender la dificultad de darle consistencia en la percepción y aplicación a los servicios de soporte - reconociéndolos como los básicos para la ocurrencia de los tres mencionados; la aplicación de estos conceptos y metodologías en la gestión sostenible tanto de sistemas naturales como de agroecosistemas presenta debilidades que tienen que ver fundamentalmente con la interpretación y apropiación del concepto a un contexto, escala y tiempo determinados y la viabilidad de lo propuesto.

De otra parte, dentro del planteamiento de la sostenibilidad como una propiedad emergente de la interacción humana para superar nuestra vulnerabilidad y cultivar prácticas que generen y sustenten la vida movilizando la imaginación (De Souza, 2012) surgen dos tendencias de la revisión antecedente: la primera acerca de la necesidad de realizar una valoración integral de los servicios ecosistémicos, reconociendo que los sistemas socioecológicos como el de la palma de aceite se incluyen como demandantes y oferentes de servicios ecosistémicos; la segunda la importancia de hacer monitoreo de los servicios ecosistémicos a escala de paisaje a lo largo del tiempo para lograr mosaicos sostenibles de uso del suelo e incorporación de las percepciones locales; también se agrega la necesidad de comparar el impacto que tienen diferentes alternativas de gestión sobre los ecosistemas y el bienestar humano a través de procesos de evaluación. En este aspecto se tendrán que trabajar el tema de gobernanza, de organización y participación de los actores 
sociales, grupos de interés y especialmente de la institucionalidad local (Martín-López et al., 2014; RincónRuiz et al., 2014).

De igual manera la alta complejidad del enfoque interdisciplinar si bien es un ejemplo de la sinergia del conocimiento disciplinar, genera dificultades en tres temas importantes: la comprensión y manejo de la incertidumbre, los métodos para identificar, cualificar y cuantificar los servicios ecosistémicos y sus relaciones con la biodiversidad (trades-offs, dis-servicios, sinergias), y el análisis particular y ajustado al contexto de los servicios ecosistémicos culturales.

En consecuencia los servicios ecosistémicos pueden convertirse en un soporte para la decisión acertada, en términos de gestión y de política (Schneiders et al., 2012; Vilardy et al., 2012; Partidario y Gomes, 2013) ante la demanda creciente de biodiversidad y sus recursos. Dada la complejidad de los sistemas socioecológicos, que permiten en una situación determinada, entender la retroalimentación entre el sistema natural y el sistema social mediada por los servicios ecosistémicos y reflejada en los beneficios, así como la condición de incertidumbre y de variaciones espaciotemporales inherentes a su funcionamiento, la propuesta de valoración integral de servicios constituye una opción apropiada siempre y cuando se analicen los servicios culturales con enfoque de no monetización y se incorporen en todo el proceso de diagnóstico y valoración a los actores locales, grupos de interesados e instituciones; la apropiación estará vinculada al carácter interdisciplinar, al contexto correspondiente con las escalas espacio temporales y con las condiciones jerárquicas seleccionadas.

\section{Referencias}

Anastasopoulou S, Chobotova V, Dawson T, Kluvankova-Oravska T, Rounsevell M. (2007). Identifying and assessing socio-economic and environmental drivers that affect ecosystems and their services. The RUBICODE Project. Rationalising Biodiversity Conservation in Dynamic Ecosystems.

Anderies JM, Janssen MA, Ostrom E. A framework to analyze the robustness of social-ecological systems from an institutional perspective. Ecology and society. 2004;9(1):18.

Andrade GI, Castro, LG. Degradación, pérdida y transformación de la biodiversidad continental en Colombia, invitación a una interpretación socioecológica. Ambiente y Desarrollo. 2012; $\mathrm{XVI}(30): 53-71$.

Andrade Gl, Wills E, Serje M, Madriñán S, Romero M, Delgado J, et al. 2014. Ecosistema empresarial agroindustrial. Proceso de construcción de un paisaje agroindustrial sostenible en el proyecto Veracruz, frontera agroindustrial de la altillanura en la Orinoquia colombiana. Centro de Estrategia y Competitividad,
No. 2. Bogotá: Universidad de los Andes, Facultad de Administración. 120 p.

Andrade-Pérez GI, Romero M, Delgado J. Diseño adaptativo de un paisaje agroindustrial. Una propuesta para la transformación agrícola de la altillanura colombiana. Ambiente y Desarrollo. 2013;17(33):29-40.

Andrade GI, Sandino JC, Aldana J. 2011. Biodiversidad y territorio. Innovación para la gestión adaptativa ante el cambio ambiental global. Bogotá: Instituto de Investigación de Recursos Biológicos Alexander von Humboldt.

Arosa RF. 2015. Aportes al mejoramiento de la calidad de vida de los migrantes vinculados a la agroindustria de palma de aceite (Elaeis guineensis) en el municipio de Maní, Casanare. Trabajo de grado para optar al título de magister en Gestión Ambiental Sostenible. Universidad de los Llanos.

Ash N, Bennett K, Reid W, Irwin F, Ranganathan J, Scholes R, et al. 2010. Assessing Ecosystems, Ecosystem Services, and Human Well-being. Chapter I. En: Ash N, Blanco H, Brown C, Garcia K, Henrichs T, Lucas N, Raudsepp-Hearne C, Simpson RD, Scholes R, Tomich TP, Vira B, and Zurek M. 2010. Ecosystems and human well-being: a manual for assessment practitioners. Library of Congress Cataloging-in-Publication Data. USA. p 19-51.

Balvanera P, Uriarte M, Almeida-Leñero L, Altesor A, DeClerck, Gardner T, et al. Ecosystem services research in Latin America: The state of the art Ecosystem Services. 2012;(2):56-70

Balvanera P, Castillo A, Avila P, Caballero K, Flores A, Galicia C, et al. 2011. Marcos conceptuales interdisciplinarios para el estudio de los servicios ecosistémicos en América Latina. El valor ecológico, social y económico de los servicios ecosistémicos. Conceptos, herramientas y estudio de casos, pp: 00.

Balvanera P, Cotler H, Aburto O, Aguilar A, Aguilera M, Aluja M, et al. 2009. Estado y tendencias de los servicios ecosistémicos. Capital natural de México, 2, 185-245.

Balvanera P, Cotler H. Acercamientos al estudio de los servicios ecosistémicos. Gaceta ecológica número especial 2007;8485(2007):8-15.

Barnaud C, Antona M. Deconstructing ecosystem services: Uncertainties and controversies around a socially constructed concept. Geoforum. 2014;56:113-123

Benavides J. 2010. El desarrollo económico de la Orinoquia, como aprendizaje y construcción de instituciones. Debates Presidenciales. CAF-Fedesarrollo.

Bennet E, Peterson GD, Gordon L. Understanding relationships among multiple ecosystem services. Ecology Letters. 2009;12:111.

Boyd J, Banzhaf SWhat are ecosystem services? The need for standardized environmental accounting units. Ecological Economics. 2007;63:616-626.

Brundtland GH.1987. Reporte del Mundo. Comisión de Ambiente y Desarrollo. Naciones Unidas, Comisión de Ambiente y Desarrollo. Estados Unidos: ONU. 
Bustamente C. 2014. Agroecosistemas. Conferencia magistral. Doctorado en Ciencias Agrarias. Producción Sostenible. Octubre de 2014. Villavicencio. Universidad de los Llanos.

Burkhard B, Kroll F, Müller F, Windhorst W. Landscapes' capacities to provide ecosystem services-a concept for land-cover based assessments. Landscape online. 2009;15(1):22.

Cardona Trujillo H. 2011. Gestión de los servicios ecosistémicos que presta la flora de los agroecosistemas de la cuenca del río La Vieja, eje cafetero, Colombia. Trabajo de grado para optar al título de magister en desarrollo rural. Pontificia Universidad Javeriana.

Carpenter SR, Mooney HA, Agard J, Capistrano D, DeFries RS, Díaz $\mathrm{S}$, et al. Science for managing ecosystem services: Beyond the Millennium Ecosystem Assessment. Proceedings of the National Academy of Sciences. 2009; 106(5):1305-1312.

Carrizosa Umaña J. 1992. La política ambiental en Colombia: desarrollo sostenible y democratización. CEREC, FESCOL, Fondo FEN Colombia. Bogotá. 220 pp.

Conabio. 2006. Capital natural y bienestar social. Comisión Nacional para el Conocimiento y Uso de la Biodiversidad, México.

Carson R. 1962. Silent spring. London: Hamish Hamilton.

Cork SJ, Peterson GD, Bennett EM, Petschel-Held G, Zurek M. Synthesis of the storylines. Ecology and Society. 2006;11(2):11

Costanza R, Kubisszewski I. The authorship structure of "ecosystem services" as a transdisciplinary field of scholarship. Ecosystem Services. 2012;1(1):16-25.

Costanza R, Arge R, de Groot R, Farber S, Grasso M, Hannon B, et al. The value of the world's ecosystem services and natural capital. Nature. 1997;387:253-260.

Costanza R, Folke C. Valuing ecosystem services with efficiency, fairness and sustainability as goals. Nature's services: Societal dependence on natural ecosystems. 1997;49-70.

Costanza R, Daly HE. Natural capital and sustainable development. Conservation biology. 1992;6(1):37-46.

Cooper RN. 1975. Natural resources and national security. Resources Policy. Abstract. Trabajo presentado en 16th Annual conference of the international Institute for Strategic Studies, Brighton England 1974.

Chee YE. An ecological perspective on the valuation of ecosystem services. Biological conservation. 2004; 120(4): 549-565.

Christie M, Fazey I, Cooper R, Hyde T, Kenter, JO. An evaluation of monetary and non-monetary techniques for assessing the importance of biodiversity and ecosystem services to people in countries with developing economies. Ecological Economics. 2012;83:67-78.

Crossman ND, Burkhard B, Nedkov S, Willemen L, Petz K, Palomo $\mathrm{I}$, et al. A blueprint for mapping and modelling ecosystem services. Ecosystem Services. 2013; 4:4-14.

Daily GC, Söderqvist T, Aniyar S, Arrow K, Dasgupta P, Ehrlich $\mathrm{PR}$, et al. The value of nature and nature of value. Science. 2000;289(5478):395-396
Daily GC. 1997. Introduction: What are ecosystem services? In Daily GC. ( ed). Natures Services: Societal Dependence on Natural Ecosystems. Island Press, Washington, D.C.

Daily GC, Alexander S, Ehrlich PR, Goulder L, Lubchenco J, Matson PA, et al. Ecosystem services: benefits supplied to human societies by natural ecosystems. Issues in Ecology. 1997;2:1-18.

Darwin C. 1872. The origin of species by means of natural selection. Sixth edition. The New American Library, New York, New York. $478 \mathrm{pp}$.

de Groot RS, Alkemade R, Braat L, Hein L, Willemen L. Challenges in integrating the concept of ecosystem services and values in landscape planning, management and decision making. Ecological Complexity. 2010;7:260-272.

de Groot RS, Wilson M, Boumans R. A typology for the description, classification and valuation of ecosystem functions, goods and services. Ecological Economics. 2002;41(3):367-567.

De Souza Silva José. "El 'día después del desarrollo en América Latina. Descolonizar la comunicación y la educación para liberar la gestión hacia la construcción de comunidades felices con modos de vida sostenibles", Brasil, Pontificia Universidad Católica del Ecuador - PUCE Escuela de Trabajo Social - Carrera en Gestión Social, 2011.

Díaz S, Fargione J, Chapin FS III, Tilman D. Biodiversity Loss Threatens Human Well-Being. PLoS Biol. 2006;4(8):e277

Douglas LR, Alie K. High-value natural resources: Linking wildlife conservation to international conflict, insecurity, and development concerns. Biological Conservation. 2014;171:270-277.

Egoh B, Rouget M, Reyers B, Knight AT, Cowling RM, Van Jaarsvel AS, Welz A. Integrating ecosystem services into conservation assessments: a review. Ecological Economics. 2007;63:714721.

Ehrlich R, Mooney HA. Extinction, Substitution, and Ecosystem Mooney Source: BioScience. 1983;33(4):248-254.

Epstein G, Vogt JM, Mincey K, Cox M, Fischer B. Missing ecology: integrating ecological perspectives with the social-ecological system framework. International Journal of the Commons. 2013;7(2):432-453.

Fernández GA. 2013. Análisis de los impactos en los servicios ecosistémicos culturales generados por la expansión del cultivo de palma africana (Elaeis guineensis) en el municipio de Villanueva, Casanare. Trabajo de grado para optar por el título de Magíster en Desarrollo Rural. Pontificia Universidad Javeriana.

Fisher B, Costanza R, Turner RK, Morling P. Defining and classifying ecosystem services for decision making. Ecological economics. 2009;68(3):643-653.

Folke C. Resilience: The emergence of a perspective for socialecological systems analyses. Global Environmental Change. 2006; 16:253-267.

García Llorente M, Martín-López B, Iniesta-Arandia I, López-Santiago C, Aguilera P, Montes C. The role multi-functionality in social preferences toward semi-arid rural landscape: An ecosystem services approach. Environmental Science \& policy. 2012;1920:136-146. 
Goebertus J. Palma de Aceite y Desplazamiento Forzado en Zona Bananera Trayectorias "Entre Recursos Naturales y Conflicto". Colombia Internacional. 2008;67(192):152-175.

Gómez-Baggethun E, de Groot R, Lomas PL, Montes C. The history of ecosystem services in economic theory and practice: from early notions to markets and payment schemes. Ecological Economics. 2010;69(6):1209-1218.

Gómez-Baggethun E, de Groot R. Capital natural y funciones de los ecosistemas: explorando las bases ecológicas de la economía. Ecosistemas. 2007;16:4-14.

González JA, Montes C, Santos I. Capital natural y desarrollo: por una base ecológica en el análisis de las relaciones NorteSur. Papeles de Relaciones Ecosociales y Cambio Global. 2008;100:63-78.

Grima APL, Horton S, Kant S. 'Introduction: natural capital, poverty and development' Environment Development and Sustainability. 2003;5:297-314.

Haines-Young R, Potschin M. The links between biodiversity, ecosystem services and human well-being. Ecosystem Ecology: a new synthesis. 2010;110-139.

Harrison PA, Vandewalle M, Sykes MT, Berry PM, Bugter R, de Bello $\mathrm{F}$, et al. Identifying and prioritising services in European terrestrial and freshwater ecosystems. Biodiversity and Conservation. 2010;19(10):2791-2821.

Hawkins K. 2003. Economic valuation of ecosystem services. University of Minnesota, 23.

Henson I, Ruiz R, Romero HM. The greenhouse gas balance of the oil palm industry in Colombia: a preliminary analysis. I. Carbon sequestration and carbon offsets. Agronomía Colombiana. 2012;30(3): 359-369.

Holdren JP, Ehrlich PR. Human Population and the Global Environment: Population growth, rising per capita material consumption, and disruptive technologies have made civilization a global ecological force. American Scientist. 1974;282-292.

Howe C, Suich H, Vira B, Mace GM. Creating win-wins from tradeoffs? Ecosystem services for human well-being: A meta-analysis of ecosystem service trade-offs and synergies in the real world. Global Environmental Change. 2014;28:263-275.

Humboldt A Von. 2011. Cosmos. Ensayo de una descripción física del mundo. Madrid-Santiago de Chile: Libros de la Catarata, Consejo Superior de Investigaciones Científicas y Dirección de Bibliotecas, Archivos y Museos (1era edición 1845-1862).

Humphreys, M. Natural resources, conflict, and conflict resolution: Uncovering the mechanisms. Journal of Conflict Resolution. 2005;49(4):508-537.

Instituto de Investigación de Recursos Biológicos Alexander von Humboldt - IAvH -.2013. Plan Estratégico de la Macrocuenca del Orinoco. Fases I y II. Informe técnico final entregado 'por' 4D'Elements'consultores. $321 \mathrm{p}$.

Janssen MA, Ostrom E. Governing social-ecological systems. Handbook of computational economics. 2006;2:1465-1509.
Janssen W, Goldsworthy P. Interdisciplinary Research for Natural Resource Management: Conceptual and Practical Implications. Agricultural Systems. 1996;51:259-279.

King A, Okita S, Peccei A, Pestel E, Thiemann H, Wilson C. Los límites del crecimiento. Informe del Club de Roma sobre el Predicamento de la Humanidad. F.C.E, 1973 (1º Edición en inglés 1972).

Kremen C. 2005. Managing ecosystem services: what do we need to know about their ecology?. Ecology Letters. 2005;8:468-479.

Labarca M, Portillo E, Portillo A, Morales A. Estructuras reproductivas y polinización entomófila en tres lotes comerciales de palma aceitera (Elaeis guineensis Jacq.) en el estado Zulia, Venezuela. Revista de la Facultad de Agronomía. 2009;26:1-22.

Lamarque P, Quétier F, Lavorel S. The diversity of the ecosystem services concept and its implications for their assessment and management. Comptes Rendus Biologies. 2011; 334(5);441-449.

Laurans Y, Mermet L. Ecosystem services economic valuation, decision-support system or advocacy?. Ecosystem Services. 2014;7:98-105.

Lavelle P, Rodríguez N, Arguello O, Berna J, Botero C, Chaparro P, et al. Soil ecosystem services and land use in the rapidly changing Orinoco River Basin of Colombia. Agriculture, Ecosystems and Environment. 2014;185:106-117.

Larraín J, Dávila, RC. 2004. Identidad y modernidad en América Latina. Oceano.

León ST, Valbuena S, Borrero M. 2006. Palma de aceite, biodiversidad y tendencias de política: el caso de la Orinoquia colombiana. Bogotá. Instituto de Investigaciones de Recursos Biológicos Alexander von Humboldt - World Wildlife Fund. Informe final, 176 p.

Lele S, Springate-Baginski O, Lakerveld R, Deb D, Dash P. Ecosystem services: origins, contributions, pitfalls, and alternatives. Conservation and Society. 2013;11(4):343.

Margalef R. 1993. Teoría de los sistemas ecológicos. Segunda edición. Universidad de Barcelona 290 p.

Linne CA. 1788. Systema naturae, per regna tria naturae, secundum classes, ordenis, genera, species, cum sharacteribus, diferentiis, sinonimys, locis. Tomus I. Impensis Georg Emanuel Beer, Leipzig, Alemania.

McAfee K. The contradictory logic of global ecosystem services markets. Development and Change. 2012;43(1):105-131.

MacDonald DV, Hanley N, Moffatt I. Applying the concept of natural capital criticality to regional resource management. Ecological Economics. 1999;29:73-87.

Mace GM, Norrisand K, Fitter. AH. Biodiversity and ecosystem services: a multilayered relationship. Trends in Ecology and Evolution. 2012;27:19-26.

Malthus TR. 1963. A summary view of the principle of population. New American Lib.

Márquez CG. 1996. Ecosistemas estratégicos y otros estudios de ecología ambiental. En: Ecosistemas estratégicos y otros estudios de ecología ambiental. 
Martínez-Alier J, Munda G, O’Neill J. Weak comparability of values as a foundation for ecological economics. Ecological economics. 1998;26(3):277-286.

Martín-López B, Gómez-Baggethun E, García-Llorente M, Montes C. Trade-offs across value-domains in ecosystem services assessment. Ecological Indicators. 2014;37:220-228.

Martín-López B, Iniesta-Arandia I, García-Llorente M, Palomo I, Casado-Arzuaga I, García Del Amo D, et al. Uncovering ecosystem service bundles through social preferences. PloS one. 2012a;7(6):e38970.

Martín-López B, González JA, Vilardy SP, Montes C, García-Llorente M, Palomo I, Aguado M. 2012b. Guía docente ciencias de la sostenibilidad. Universidad del Magdalena, el Instituto Humboldt y la Universidad Autónoma de Madrid. 146 pp.

Martín-López B, Montes C. 2011. Biodiversidad y servicios de los ecosistemas. Observatorio de la Sostenibilidad en España (OSE). 444-465.

Martín-López B, Gómez-Baggethun E, Montes C. Un marco conceptual para la gestión de las interacciones naturaleza-sociedad en un mundo cambiante. Cuaderno Interdisciplinar de Desarrollo Sostenible - CUIDES. 2009;3:229-258.

Maskell LC, Crowe A, Dunbar MJ, Emmett B, Henrys P, Keith AM, et al. Exploring the ecological constraints to multiple ecosystem service delivery and biodiversity. Journal of Applied Ecology. 2013;50(3):561-571.

Millenium Ecosystem Aseessment - MEA -. 2003. Ecosystem and human well-being: A framework for assessment. Island Press. Washington. D.C.

Millenium Ecosystem Assessment - MEA -. 2005. Ecosystem and human well-being: A framework for assessment. 4 volumes. Island Press. Washington. D.C, EE.UU.

Ministerio de Ambiente y Desarrollo Sostenible - MADS. 2012. Política nacional para la gestión integral de la biodiversidad y sus servicios ecosistémicos. República de Colombia. 134 p.

Mora-Fernández C, Peñuela-Recio L. (Eds.) 2013. Salud Ecosistémica de las sabanas inundables asociadas a la cuenca del río Pauto, Casanare, Colombia. Yoluka ONG, fundación de investigación en biodiversidad y conservación, Fundación Horizonte Verde y Ecopetrol S.A. 150pp.

Mosquera M, García E. Impacto social de la agroindustria de palma de aceite. PALMAS. 2005;26(2):11-19.

Montes C. Del Desarrollo Sostenible a los servicios de los ecosistemas. Ecosistemas. 2007;16(3):1-3.

Motesharrei S, Rivas J, Kalnay E. Human and nature dynamics (HANDY): Modeling inequality and use of resources in the collapse or sustainability of societies. Ecological Economics. 2014;101:90-102.

Nelson GC, Bennett E, Berhe AA, Cassman K, DeFries R, Dietz T, et al. Ecosystem services: From eye-opening metaphor to complexity blinder. Ecological economics. 2010; 69(6):1219-1227.

Ochoa AJ, Chávez HE. 2010. Evaluación de la sostenibilidad social y económica en los cultivos de palma africana en el departa- mento del meta. Propuesta de trabajo de grado para optar al título de Magister en Desarrollo Sostenible y Medio Ambiente. Universidad de Manizales.

Odum EP. The strategy of Ecosystem development. Science. 1969;164(3877):262-270.

Ojima D, Pingali P, Watson R, Zurek M. Anthropogenic drivers of ecosystem change: an overview. Ecology and Society. 2006;11(2):29.

Organización de Naciones Unidas - ONU -. 1992. Informe de la Conferencia de las Naciones Unidas sobre el Medio Humano. Estocolmo. S.73.II.A.14. Disponible en http://www.un.org/spanish/esa/sustdev/documents/declaracionrio.htm

Organización de Naciones Unidas - ONU -. 2002. Cumbre Mundial sobre el desarrollo sostenible. Johannesburgo. Disponible en http://www.un.org/spanish/conferences/wssd/

Organización de Naciones Unidas - ONU-. 2012. Conferencia de las Naciones Unidas sobre el Desarrollo Sostenible. El futuro que queremos. Rio de Janeiro. Consultado en http://www. un.org/es/sustainablefuture/about.shtml.

Ostrom E. A General Framework for Analyzing Sustainability of Social-Ecological Systems. Science. 2009;325(5939):419-422.

Partidario MR, Gomes RC. Ecosystem services inclusive strategic environmental assessment. Environmental Impact Assessment Review. 2013;40:36-46.

Perrings CA. 1994. Biotic diversity, sustainable development, and natural capital. Investing in natural capital: the ecological economics approach to sustainability. Washington, DC: Island Press. 92-112p.

Prugh T, Costanza R, Cumberland J, Herman E, Daly R, Goodland R, Norgaard B. 1999. Natural Capital And Human Economic Survival. Second edition. CRC Press

Raudsepp-Hearne C, Peterson GD, Tengö M, Bennett EM, Holland $\mathrm{T}$, Benessaiah $\mathrm{K}$, et al. Untangling the environmentalist's paradox: why is human well-being increasing as ecosystem services degrade?. BioScience. 2010;60(8):576-589.

Reyers B, Polasky S, Tallis H, Mooney HA, Larigauderi, A. Finding Common Ground for Biodiversity and Ecosystem Services. BioScience. 2012;62(5):503-507.

Rincón-Ruíz A, Echeverry-Duque M, Piñeros AM, Tapia CH, David A, Arias-Arévalo P, Zuluaga PA. 2014. Valoración integral de la biodiversidad y los servicios ecosistémicos: Aspectos conceptuales y metodológicos. Instituto de Investigación de Recursos Biológicos Alexander von Humboldt (IAvH). Bogotá, D.C. Colombia. 151 pp.

Rivera-Pabón JA. Paisajes alterados: retrospectiva del manejo ecológico social de la vertiente. Cordillera central. Eje cafetero colombiano. Luna Azul. 2008;(27):27-48.

Rivera L, Ambrecht I. Diversidad de tres gremios de hormigas en cafetales de sombra y sol y bosque de Risaralda. Revista Colombiana de Entomología. 2005;31(1):89-96.

Rodríguez JP, Beard TD, Bennett EM, Cumming GS, Cork S, Agard J, Dobson AP, Peterson GD. Trade-offs across space, time, and ecosystem services. Ecology and Society. 2006;11(1):28. 
Rodríguez-Labajos B, Martínez-Alier J. The economics of ecosystems and biodiversity: recent instances for debate. Conservation and Society. 2013;11(4):326.

Rositano F, López M, Benzi P, Ferraro DO. Servicios de los ecosistemas: un recorrido por los beneficios de la naturaleza. Rev. Agronomía \& Ambiente. 2012;32(1-2):49-60.

Ruiz JP. Biocombustibles, medio ambiente y desarrollo social. Revista Javeriana. 2007; 738:52-59.

Sarandón SJ, Flores CC. 2009. Evaluación de la sustentabilidad en agroecosistemas: Una propuesta metodológica.

Schumacher EF. 1973. Small is Beautiful: Economics as if People Mattered. Blond and Briggs, London. 288 pp

Sanabria C, Lavelle P, Fonte SJ. Ants as indicators of soil-based ecosystem services in agroecosystems of the Colombian Llanos. Applied Soil Ecology. 2014;84:24-30.

Schneiders A, Van Daele T, Van Landuyt W, Van Reeth W. Biodiversity and ecosystem services: Complementary approaches for ecosystem management? Ecological Indicators. 2012;21:123133.

Skourtos M, Kontogianni A, Harrison PA. Reviewing the dynamics of economic values and preferences for ecosystem goods and services. Biodiversity and conservation. 2010;19 (10):2855-2872.

Spangenberg JH, Settele J. Precisely incorrect? Monetising the value of ecosystem services. Ecological Complexity. 2010;7(3):327337.

Spash CL, Aslaksen I. 2012. Re-establishing an Ecological Discourse in the Debate over the Value of Ecosystems and Biodiversity. SRE - Discussion Papers, 2012/05. WU Vienna University of Economics and Business, Vienna.

Syrbe RU, Watz U. Spatial indicators for the assessment of ecosystem services: Providing, benefiting and connecting areas and landscape metrics. Ecological Indicators. 2012;21(2012):8088.

Tapella E. 2012. Heterogeneidad social y valoración diferencial de servicios ecosistémicos. Un abordaje multi-actoral en el oeste de Córdoba (Argentina). Tesis de doctorado para optar al grado académico de doctor en Ciencias Agropecuarias. Universidad Nacional de Córdoba. Facultad de Ciencias Agropecuarias Escuela para Graduados. 367 páginas.

The economics of ecosystems and biodiversity - TEEB. 2010. The Economics of Ecosystems and Biodiversity: Ecological and Economic Foundations, Earthscan, London and Washington.

Torres DC, Baptiste B y Caro C. Propuesta de gestión territorial del complejo ecológico asociado con la laguna Carimagua, a partir de una evaluación de servicios ecosistémicos: municipio de Puerto Gaitán-Meta. Ambiente y Sostenibilidad. 2014;(4):7988.

Trabucchi M, Ntshotsho P, O'Farrell P, Comín FA. Ecosystem service trends in basin-scale restoration initiatives: a review. Journal of environmental management. 2012;111: 18-23.

Troy A, Wilson M. Mapping ecosystem services: practical challenges and opportunities in linking GIS and value transfer. Ecological Economics. 2006;60:435-449.

Van den Belt $M$, Blake D. Ecosystem services in new Zealand agro-ecosystems: A literature review. Ecosystem Services. 2014;9:115-132.

Vandewalle M, Sykes MT, Harrison PA, Luck GW, Berry P, Bugter R, et al. 2008. Concepts of dynamic ecosystems and their services. The RUBICODE Project. Rationalising Biodiversity Conservation in Dynamic Ecosystems.

Vallés-Planells M, Galiana F, Van Eetvelde V. A classification of landscape services to support local landscape planning. Ecology and Society. 2014;19(1):44.

Vilardy S, González JA, Martín-López B, Oteros-Rozas E, Montes C. Los servicios de los ecosistemas de la Reserva de Biosfera Ciénaga Grande de Santa Marta. Revista Iberoamericana de Economía Ecológica. 2012;19:66-83.

World Trade Organization- WTO- . 2010. World Trade Report 2010 Trade in natural resources. Online WTO bookshop: http://onlinebookshop.wto.org. 46 p. 